\title{
Spatial Resolution of Scanning Electron Microscopy without a Vacuum Chamber
}

\author{
Kayla Nguyen ${ }^{1}$, Justin Daniel Richmond-Decker ${ }^{2}$, Megan Holtz ${ }^{2}$, Yonat Milstein ${ }^{3}$, David A. Muller ${ }^{2}$ \\ ${ }^{1}$ Department of Chemistry and Chemical Biology, Cornell University, Ithaca, NY, USA. \\ ${ }^{2}$ School of Applied and Engineering Physics, Cornell University, Ithaca, NY, USA. \\ ${ }^{3}$ b-Nano Ltd, Rehovot, Israel.
}

From its initial widespread availability almost half century ago, the scanning electron microscope (SEM) has become a ubiquitous tool for metrology, research and development [1]. Modifications to the SEM such as environmental scanning electron microscopy (ESEM) [2] have enabled imaging of specimens in gaseous environment through pressures limiting apertures. In addition to ESEM, Atmospheric-SEM (ASEM) [3] uses an inverted geometry for imaging specimens in liquid or at atmospheric pressures in contact with an electron-transparent window. More recently, the airSEM [4], a field emission SEM that operates without a vacuum chamber, allows a rapid exchange of samples without having to replace the atmospheric window. The airSEM uses an upright geometry with an electron transparent windowseparating vacuum from air (Fig 1a). The samples are loaded by computer-controlled optical alignment. The accessibility of the sample, without the need for vacuum feedthroughs makes it very simple to add imaging modes, including secondary ion detector, $\mathrm{x}$-ray mapping, cathodoluminescence, and correlative optical and ultrafast imaging. Here we show that nanoscale resolution can be retained at atmospheric gas path lengths (GPL) as long as $400 \mu \mathrm{ms}$, and that beam spreading in the electron-transparent window is the dominant effect on contrast at typical operating conditions.

High-resolution imaging is possible as long as a usable fraction of the incident beam remains unscattered, and the scattered electrons are so dispersed as to provide a broad, structureless background, as can be seen in the Monte Carlo simulations [5] of Fig 1b. The contrast reduction will then follow a product of Beer's law exponential decays for the window, and the gas atmosphere. The key is then to ensure that the window and GPL thickness should not exceed a few mean free paths (mfp). The $\mathrm{mfp}$ for the window is $\sim 30 \mathrm{~nm}$ for typical material, and $75-600 \mu \mathrm{ms}$ for air and typical air-He mixtures at 30 $\mathrm{keV}$. Monte Carlo simulations of bright-field STEM contrast for a $50 \mathrm{~nm}$ Au nanoparticle (Fig 1c) support this simple analytic model, and suggest a x2.5 increase in contrast by reduction the SiNx window thickness from 30 to $5 \mathrm{~nm}$. Experimental airSTEM, images are taken at increasing gas path lengths (Fig 2a,b). Measured and simulated line profiles (Fig 2c,d) show nanoscale edge resolution is retained even at a 400 um gas path length in an air-He mixture.

For SEM imaging, the contrast and resolution of backscatter electron imaging (BSE) is improved by lowering the beam voltage. By using thinner electron transparent windows of $5 \mathrm{~nm}$, lower beam energy $(7 \mathrm{keV})$ becomes practical. Fig $3 \mathrm{a}$ shows BSE image of wet moss. Ionization of gas molecules produces measurable ion current, allowing a form of gas amplification imaging (Fig 3b), that produces a surface sensitive signal in air. The addition of an $\mathrm{x}$-ray detector allows for the rapid mapping of samples in air, as illustrated by the BSE (Fig 3c) and x-ray compositional maps (Fig 3d) of biochar/soil mixture.[6]

References:

[1] Oliver C. Wells, Scanning Electron Microscopy. Mcgraw-Hill, 1974. 
[2] D. Stokes, Principles and Practice of Variable Pressure/Environmental Scanning Electron Microscopy. Wiley and Sons, West Sussex, (2008).

[3] M. Suga, C. Sato, et al., Ultramicroscopy 111 (2011) 1650-1658.

[4] b-Nano: http://www.b-nano.com/

[5] D. Joy, Monte Carlo Modeling for Electron Microscopy and Microanalysis. Oxford University Press, NY, (1993).

[6] Work supported by the Energy Materials Center at Cornell, DOE EFRC BES (DE-SC0001086).

Facility support from NSF MRSEC program (DMR 1120296) and the Kavli Institute at Cornell.
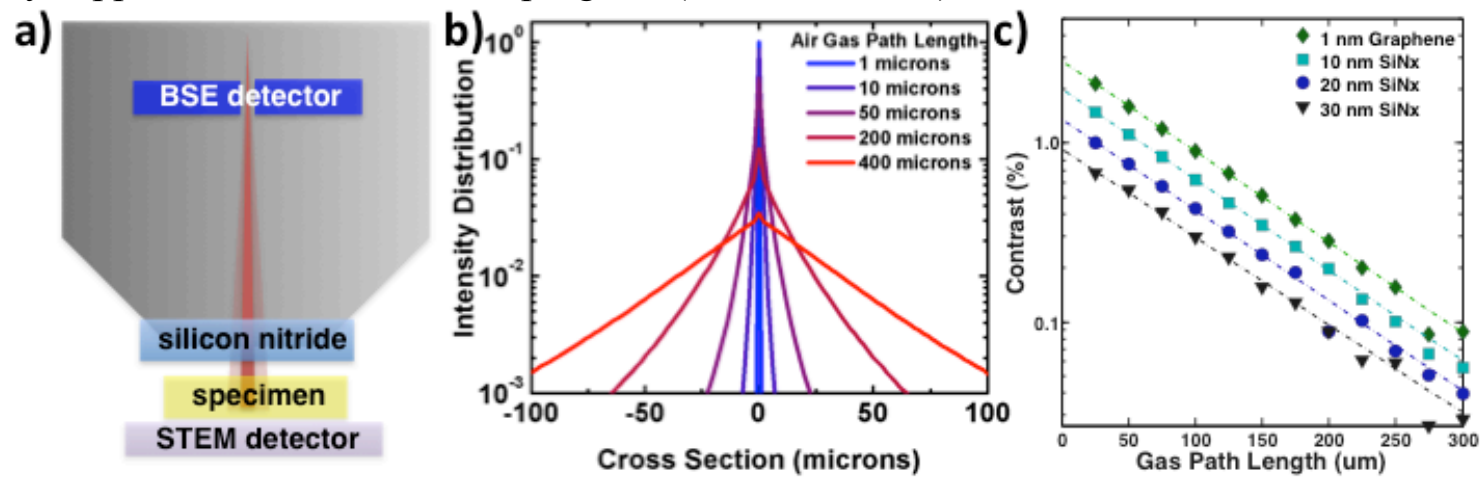

Figure 1. a) Schematic of the airSEM. b) Monte Carlo simulation of $30 \mathrm{keV}$ electron beam profiles in air. c) Monte Carlo simulation of the expected contrast for a $50 \mathrm{~nm} \mathrm{Au}$ particle through different electron-transparent windows and as a function of gas path length through air.

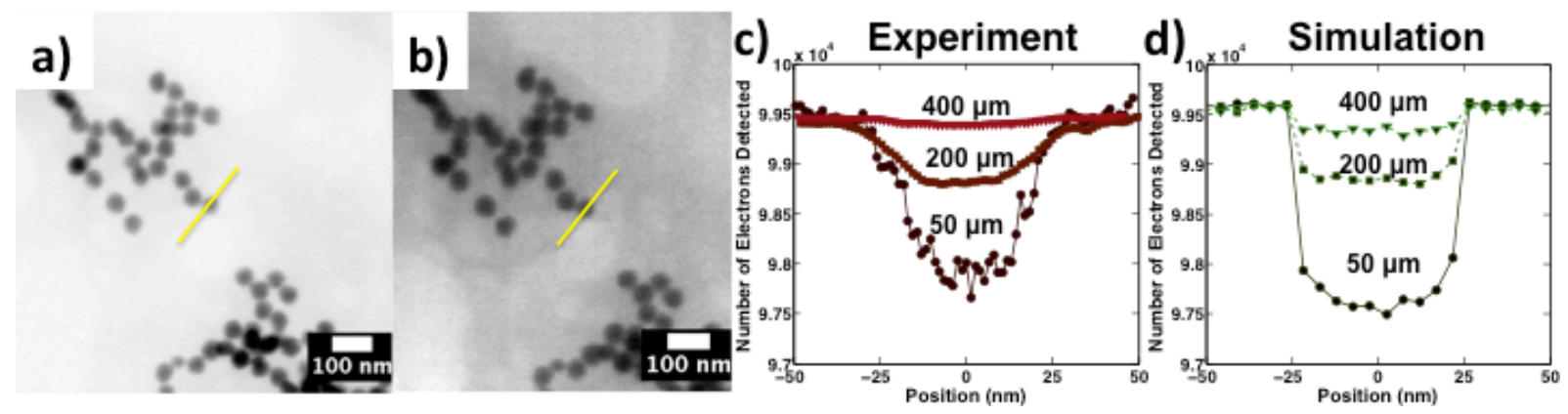

Figure 2. a) airSTEM image of Au nanoparticles at gas path lengths of $50 \mu \mathrm{m}$. b) $400 \mu \mathrm{m}$. c) Line profiles through Au nanoparticles at gas path lengths of 50, 200, $400 \mu \mathrm{m}$ taken by airSTEM. d) Monte Carlo simulation of Au nanoparticles at gas path lengths of 50, 200, $400 \mu \mathrm{m}$ through a He-air mixture.

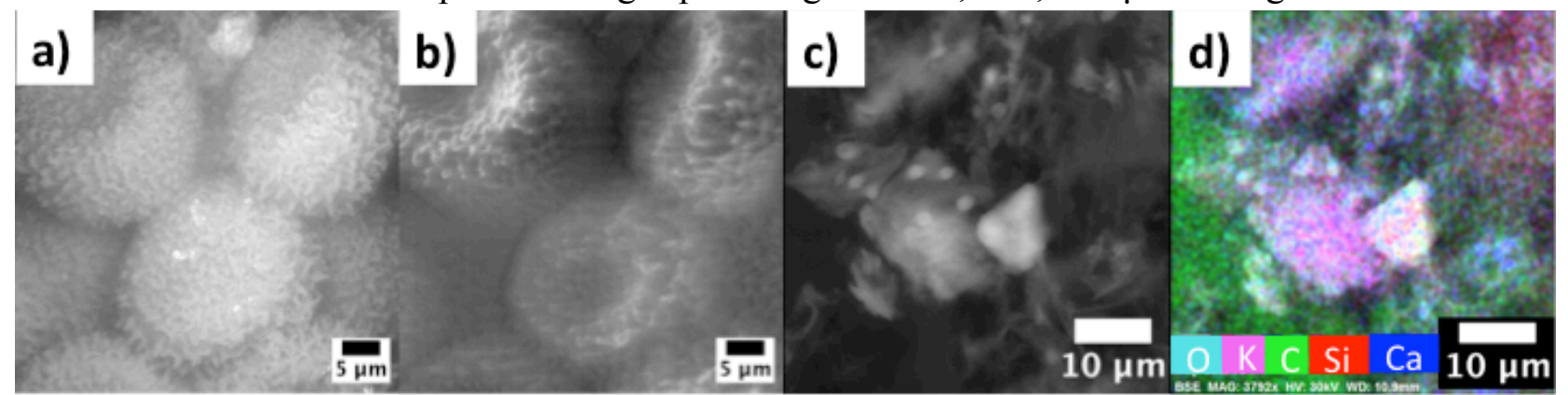

Figure 3. a) Backscattered images of wet moss in air at $7 \mathrm{keV}$ with a $5 \mathrm{~nm}$-thick SiNx window. b) Simultaneous Secondary ion current image of wet moss. c) Backscattered image of biochar at $30 \mathrm{keV}$. d) EDS map of biochar in (c). 